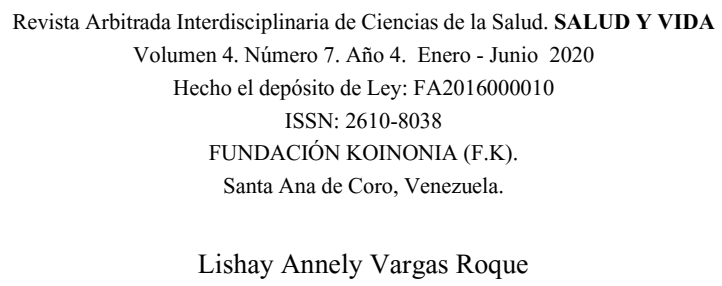

http://dx.doi.org/10.35381/s.v.v4i7.644

\title{
El Sistema Público Nacional de Salud desde la Perspectiva de la Interseccionalidad en la Línea de Frantz Fanón
}

\section{The National Public Health System from the Perspective of Intersectionality on the Frantz Fanón Line}

\author{
Lishay Annely Vargas Roque \\ lishayavr@gmail.com \\ Universidad Nacional Experimental Francisco de Miranda, Santa Ana de Coro \\ Venezuela \\ https://orcid.org/0000-0002-8104-7622
}

Recibido: 10 de octubre de 2019

Aprobado: 15 de diciembre de 2019

\section{RESUMEN}

En la investigación se utilizó el Paradigma Indígena de Investigación, el dialogo intercultural y se aplicó en el Sistema Público Nacional de Salud desde la perspectiva la Interseccionalidad en la Línea de Frantz Fanón. Conclusión: Las Instituciones de Salud occidental y la Misión Barrio Adentro manejan las mismas políticas y programas de salud. La Medicina de las instituciones de salud oficiales tiene enfoque occidental, colonial, hegemónico, curativa, biologicista, capitalista y mercantilista. La Misión Barrio Adentro, es vista por las instituciones occidentales como un subsistema paralelo. El Sistema Público Nacional de Salud esta fraccionado en subsistemas, dividido, cada institución va por su lado con sus lineamientos. La medicina tradicional se expresa con sus prácticas realizadas por los boratios, curanderos, los Homeópatas y por la medicina china. Estamos llamados a un cambio de actitud ante las posiciones de occidente, a descolonializarnos, a tener una cosmovisión de la pluriversidad y la interculturalidad en salud.

Descriptores: Salud; sistema público nacional de salud; Interseccionalidad; línea de Frantz Fanón. 


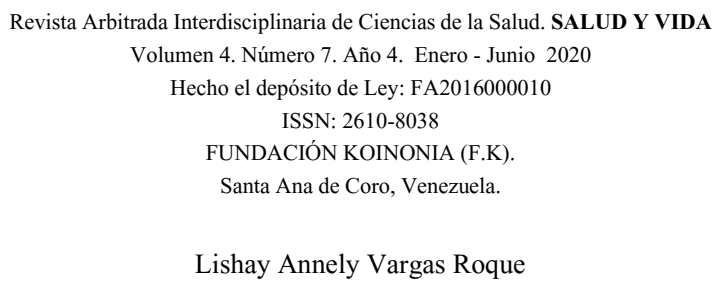

\begin{abstract}
In the investigation it was used on the Indigenous Research Paradigm, the intercultural dialogue and it was applied in the National Public Health System from the perspective of Intersectionality in the Frantz Fanón Line. Conclusion: The Western Health Institutions and the Barrio Adentro Mission handle the same health policies and programs. The Medicine of the official health institutions has a Western, colonial, hegemonic, curative, biological, capitalist and mercantilist approach. The Barrio Adentro Mission is seen by Western institutions as a parallel subsystem. The National Public Health System is divided into subsystems, divided, each institution goes along with its guidelines. Traditional medicine expresses itself with its practices carried out by the boratios, healers, the Homeopaths and by Chinese medicine. We are called to a change of attitude towards the positions of the West, to decolonialize ourselves, to have a worldview of multiculturalism and interculturality in health.
\end{abstract}

Descriptors: Health; National public health system; Intersectionality; Frantz Fanón Line.

\title{
A MODO DE INTRODUCCIÓN
}

En Venezuela el Sistema Público Nacional de Salud es fraccionado, dividido en varios subsistemas, en la cual cada institución va por su lado, con sus propios lineamientos de salud. Las prácticas que se desarrollan en las Instituciones de Salud tienen un enfoque occidental, con la creación de las nuevas políticas de salud a través de la Misión de Barrio Adentro en el 2003, ambas aplican las mismas políticas de atención de salud en niveles de atención I, II,III y IV. A diferencia de la Misión Barrio Adentro que incluye en sus programas el deportivo y la Misión Milagro, enfocado en lo preventivo.

Por otro lado, el Sistema Público Nacional de Salud es de tipo occidental, colonial, hegemónico, eurocéntrico, Biologicista, bajo el poder de las transnacionales de la Industria Farmacéutica, capitalista, mercantilista, curativa y de tipo preventiva. En la Misión Barrio Adentro, encontramos una medicina humanista, con una visión otra de la salud basada en la Atención Primaria de la Salud, con la participación del poder popular, en pro de su salud en lo familiar, comunitario y en lo social, pero con el tiempo estas políticas han venido decayendo a causa de la crisis política, social, económica del país. En Venezuela existe una pluriversidad en salud y es pertinente la integración de las Instituciones de Salud con una visión distinta a la occidental, a través de la Misión Barrio 


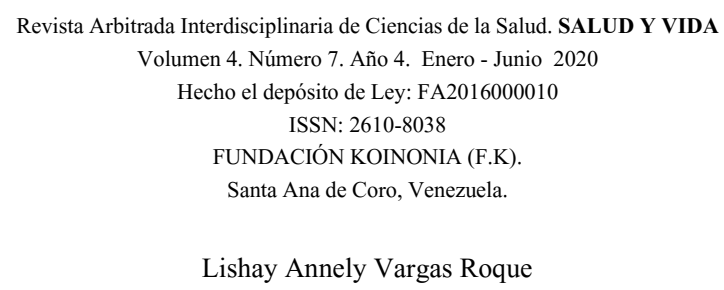

Adentro, la medicina autóctona, Homeopática, de los boratios, la medicina china, la natural y la ancestral. Es necesario que las personas que dirigen estas instituciones se encuentren con las comunidades para la construcción de ese Sistema Público Único Nacional de Salud, como verdadero sistema único.

El Sistema Público Nacional de Salud en Venezuela y en el Estado Falcón en la realidad está dividido en subsistemas, cada institución va por su lado. A pesar que existe un solo Ministerio del Poder Popular para la Salud y que los lineamientos son los mismos, no existe un único sistema. En este sistema las Instituciones de Salud tradicional califican a la Misión Barrio Adentro como un subsistema paralelo a las Instituciones de Salud occidental, la cual viene a apoderarse de los espacios en los centros de salud y a sustituir al médico venezolano. Las instituciones de salud occidental, manifiestan estar por encima de la Misión Barrio Adentro y de las personas que están en las comunidades con sus prácticas de la medicina tradicional.

Por otro lado, la definición que se tiene de la Salud en la Institución de Salud es con una visión occidental, en cambio desde la Misión de Barrio Adentro la salud es definida desde lo cultural y de la calidad de vida. En la pluriversidad y la Interculturalidad en salud, permite dar un giro a la salud con una mirada otra, con otro horizonte, en la cual se promueva el respeto a estas prácticas, es importante que se encuentren, se integren en pro de la salud de los pueblos. Es necesario el reconocimiento de los saberes de la medicina tradicional como: La de boratios, curanderos, la natural, la Homeopática, la medicina china, con la progresiva apertura de las Instituciones de Salud occidental, a esas nuevas formas para la sanación de los pueblos a través de las plantas, cremas, jarabes, semillas y brebajes elaborados de acuerdo a las enfermedades de las personas que consultan este tipo de medicina tradicional. Así como también, integrarse a la medicina de la acupuntura, de las terapias con Laser, el Biomagnetismo y a través del acercamiento a Dios por medio de la oración, el perdón; eliminando la culpa y el rencor, la sanación de las personas desde un pensamiento positivo y desde una convicción de que las enfermedades son reversibles. 


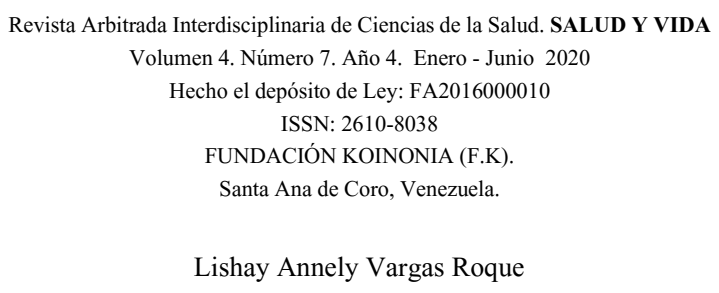

Las personas consultan tanto las instituciones de medicina occidental y la medicina tradicional. Los sitios donde se desarrollan las prácticas de la medicina tradicional es consultada por ser menos costosa, las personas han sanado, mejorado la salud, son escuchados y atendidos con buen trato. En las instituciones de medicina occidental las personas no reciben un buen trato en algunos casos. La situación política, económica y social ocasionado en Venezuela por una "guerra de V generación", han conducido a las personas a optar por otras alternativas para mejorar la salud, en el caso particular por la medicina tradicional. Es importante resaltar que en el Sistema Público Nacional de Salud específicamente en el Estado Falcón, la salud debe ser vista desde lo cultural para lograr la efectividad en la sanación de las personas. Es necesario visibilizarla partiendo de la cultura de los pueblos, con sus creencias, mitos, costumbre, saberes ancestrales, en lo autóctono y natural.

En relación a la Misión Barrio Adentro de tipo preventiva, aplica la Atención Primaria de Salud, integrando el poder popular al empoderamiento de su propia salud. Sin embargo, esta Misión Barrio Adentro es rechazada por quienes dirigen y/o están en las Instituciones de Salud occidental. Las personas que realizan las prácticas de la medicina tradicional están influenciadas unas por la religión católica y por la cristiana evangélica, así como también, las instituciones de salud occidentales están influencias por la religión. En relación a la interseccionalidad según Crenshaw (1989), es la expresión de un sistema complejo de estructuras de opresión múltiples y simultáneas, con el fin de denotar las diversas formas en que la raza y el género se intersectan para dar forma a complejas discriminaciones del sexo femenino de piel negra. Es una herramienta conceptual y analítica útil para comprender y responder a las formas en que el género se cruza con otros ejes de diferenciación como la raza, la clase social, la orientación sexual, diversidad funcional, entre otros, que dan origen a situaciones específicas de opresión o privilegio que no pueden ser comprendidas por separado. El análisis feminista de la interseccionalidad se caracteriza principalmente por ser un descentramiento del sujeto, del feminismo, al denunciar la perspectiva sesgada propuesta por el feminismo 


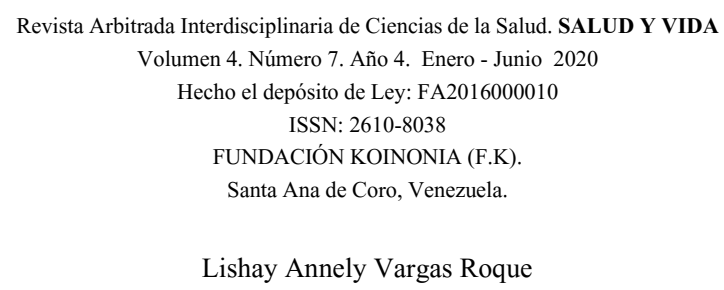

hegemónico o blanco que promoviendo la idea de una identidad común para todas las mujeres, invisibilizó a aquellas otras mujeres de color y no pertenecientes a la clase social dominante. (p. 267).

\section{DESARROLLO}

La interseccionalidad que se ha posicionado como una de las perspectivas para pensar el sujeto del feminismo, ha sido cuestionada y complejizada por el feminismo decolonial para proponer nuevos desafíos al cómo pensar el análisis interseccional y las relaciones de poder que estructuran la realidad social. Esta apuesta teórico-epistemológica que se plantea como contra hegemónica es integrada al proceso de investigación porque lleva a nuevos límites las reflexiones sobre cómo opera el poder en diversos espacios sociales, ampliando el arco de posibilidades para pensar tanto la crítica y la praxis feminista, como la investigación social, lo que ayuda a complejizar el entendimiento sobre la institucionalidad del Estado y del discurso político que da origen a las políticas públicas. Por su parte, Frantz Fanón (2010), uno de los grandes ideólogos de los movimientos de la decolonización francés, a través de su trabajo de psiquiatría elaboró un Modelo de la alienación del Colonizado. Un hombre negro que a partir de esa condición o construcción racial, analizó cómo la situación mundial degrada y condena no sólo la negritud, sino a todo aquel que representa debilidad e inferioridad desde el punto de vista hegemónico o dominante.

En cuanto a la concepción del racismo para Fanón (2010) es una Jerarquía global de superioridad sobre la Línea de lo Humano que ha sido políticamente producida y reproducida durante siglos por: Sistema Imperialista/Occidental O céntrico/Capitalista/Patriarcal/Moderno/Colonial (Grosfoguel, 2011). Las personas que están arriba de la Línea de lo Humano son reconocidas socialmente como seres humanos con derecho y acceso a subjetividad, con derechos humanos/ciudadanos/civiles/laborales. En el caso de las personas que están por debajo 


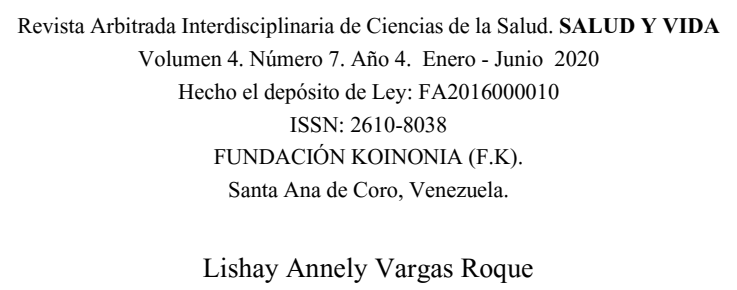

de la Línea de lo humano son consideradas sub-humanos o no humanos, es decir, su humanidad está cuestionada y negada. (Fanón, 2010, p.7).

Dependiendo de las diferentes historias coloniales en diversas regiones del mundo, la jerarquía de superioridad/inferioridad sobre la Línea de lo humano puede ser constituida con categorías raciales diversas. El racismo puede marcarse por el color, etnicidad, lenguaje, cultura y religión; es una jerarquía de superioridad/inferioridad sobre la línea de lo humano y se construye de diversas formas. Las élites occidentalizadas del tercer mundo (Africana, Asiática y Latinoamericana), reproducen prácticas racistas contra grupos etno-raciales inferiorizados donde dependiendo de la historia local/colonial la inferiorización puede ser definida o marcada a través de líneas religiosas, éticas, culturales, políticas y de color de piel. Para Fanón (2010), los sujetos localizados en el lado superior de la línea de lo humano viven en la Zona del Ser, mientras que los sujetos que viven en el lado inferior de esta línea viven en la Zona del NO Ser.(p.98).

En relación a la interseccionalidad diferenciada: Zona del Ser y de la Zona del No Ser, el mundo imperial/capitalista/colonial, la raza constituye la línea divisoria transversal que atraviesa las relaciones de opresión de clase, sexualidad y género a escala global, esto es Ilamado colonialidad del poder. (Quijano, 2000). En la Zona del Ser los sujetos por razones de ser racionalizados como seres superiores, no viven opresión racial sino privilegio racial. En cambio en la Zona del No Ser, debido a que los sujetos son radicalizados como inferiores, ellos viven opresión racial en lugar de privilegio racial. La opresión de clase, sexualidad y género que se vive en la Zona del No Ser es cualitativamente distinta a como estas opresiones se viven en la Zona del Ser. Lo que se busca enfatizar es que hay una diferencia cualitativa entre cómo las opresiones interseccionales se viven en la Zona del Ser y la Zona del No Ser en el sistema mundo capitalista/patriarcal/occidental/céntrico/cristianocentricomoderno/colonial (Grosfoguel,2011,p.98).

Las Zonas son heterogéneas tanto la del ser como la del no ser; en la Zona del Ser existen conflictos continuos entre lo que la dialéctica helegiana caracteriza como el Yo y 


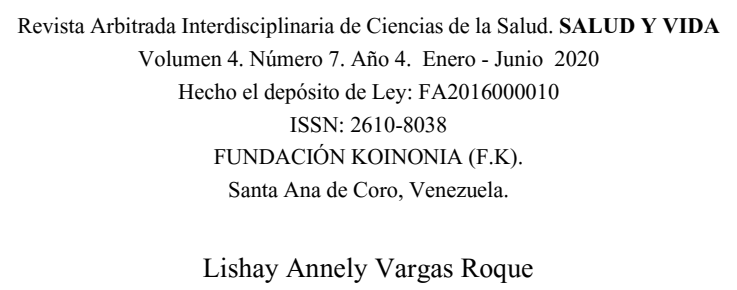

el Otro. En la dialéctica del Yo y el Otro dentro de la Zona del Ser existen conflictos que no son radicales porque la humanidad del otro oprimido es reconocida por el Yo opresor. El Yo en un Sistemalmperialista/Capitalísta/Patrialcal son las élites metropolitanas masculinas heterosexuales occidentales y las élites periféricas masculinas heterosexuales occidentalizadas. Existe un colonialismo interno tanto en el centro como en la periferia, el otro son las poblaciones occidentales de los centros metropolitanos $u$ occidentalizados dentro de una periferia cuya humanidad es reconocida, pero que al mismo tiempo viven opresiones no-raciales de clase, sexualidad o género dominados por el Yo imperial en sus respectivas regiones y países.

La Zona del Ser y del No Ser, no es un lugar geográfico específico sino una posicionalidad en relaciones radicales de poder que ocurre a escala global entre centros y periferias, pero que también ocurren a escala nacional y local contra diversos grupos radicalmente inferiorizados. Existen Zonas del Ser y No Ser a escala global entre centros occidentalizados y periferias no occidentales (colonialidad global), pero también existen zonas del ser y no ser tanto al interior de los centros metropolitanos como también dentro de la periferias (colonialismo interno). La Zona del No Ser dentro de un país sería la Zona del Colonialismo interno.

En cuanto a la posición De Sousa Santos (2010), en la modernidad existe una línea abismal entre los habitantes encima de esta línea, se puede enriquecer el entendimiento de la modernidad y su sistema mundo capitalista/imperial/patriarcal/racial-colonial que habitamos. Para Sousa (2010), la manera como se gestionan los conflictos en la Zona del Ser (arriba de la línea abismal) es a través de lo que él llama mecanismos de regulación y emancipación. Existen códigos de derechos civiles/humanos/laborales, relaciones de civilidad, espacios de negociaciones y acciones políticas que son reconocidas al otro oprimido en su conflicto con el yo dentro de la Zona del Ser. La emancipación se refiere a conceptos de libertad, autonomía e igualdad que forman parte de los fines discursivos, institucionales y legales de la gestión de los conflictos de la Zona 


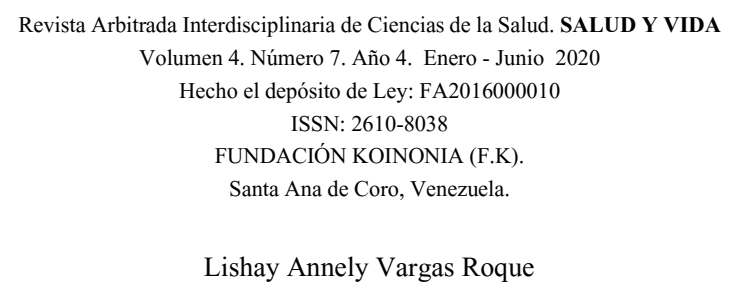

del Ser. Los conflictos en la Zona del Ser son regulados a través de métodos noviolentos.(p.99).

Por otro lado De Sousa (2010), refiere que en la Zona del No Ser de la línea abismal, donde las poblaciones son deshumanizadas en el sentido de ser consideradas por debajo de la línea de lo humano, los métodos usados por el Yo imperial/capitalista/masculino/heterosexual y su sistema institucional para gestionar y administrar los conflictos es por medio de la violencia y apropiación abierta y descarada. Los conflictos en la Zona del No Ser son gestionados por la violencia perpetua y solamente en momentos excepcionales se usan métodos de regulación y emancipación. Dado que la humanidad de la gente clasificada en la Zona del No Ser no es reconocida, porque son tratados como no humanos, sin normas de derecho y civiles, entonces se permiten actos de violencia, violaciones y apropiaciones que en la Zona del Ser serían inaceptables. Para Fanón (2010), la dialéctica del reconocimiento del yo y el otro que existe en la Zona del Ser, colapsa, se desploma en la Zona del No-Ser al no haber reconocimiento de la humanidad del otro. (p.99).

Por otro lado la interseccionalidad y la estratificación en las Zonas marcadas por la línea abismal, la opresión de clase, género y sexualidad vivida dentro de la Zona del Ser y de la Zona del No Ser son iguales. Los conflictos de las clases y élites dominantes en la Zona del Ser son de naturaleza no racial, en los conflictos de clase, género y sexualidad el "ser otro" comparte los privilegios del orden de derechos imperiales, los discursos emancipadores de la ilustración y los procesos de negociación y resolución de conflictos. Por el contrario, como en la Zona del No Ser los conflictos de clase, género y sexualidad son al mismo tiempo articulados por la opresión racial, los conflictos son gestionados y administrados con métodos violentos y de apropiación continua. La opresión de clase, sexualidad y género vivida por el No Ser Otro, es agravada debido a la articulación de dichas opresiones.

Fanón y De Sousa refieren que la Zona del Ser es el mundo imperial que incluye no solamente a las élites imperiales, sino también sujetos occidentales, mientras que la 


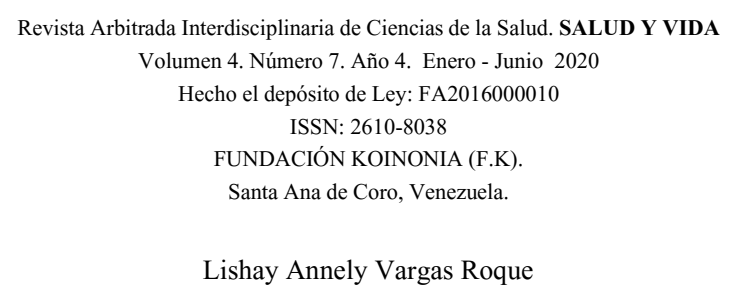

Zona del No Ser es el mundo colonial con sus sujetos oprimidos occidentales. Para ambos autores la Zona del No Ser es heterogénea y estratificada, es decir, que en la Zona del No Ser además de la opresión que los sujetos viven de parte de los sujetos en la Zona del Ser, hay también opresiones ejercidas dentro de la Zona del No Ser entre los sujetos pertenecientes a dicha zona del No Ser vive privilegios oprimiendo mujeres heterosexuales y/o gays /lesbianas no occidentales dentro de la Zona del No Ser.

A pesar de que el hombre heterosexual no occidental es un oprimido en la Zona del No Ser en relación con la Zona del Ser, la situación social es todavía peor para una mujer o un gay/ lesbianas no occidentales en la Zona del No-Ser son no solamente oprimidos por los pueblos occidentales que habitan en la Zona del Ser, sino también por otros sujetos pertenecientes a la Zona del No -Ser. Esto último, implica una doble, triple o cuádruple opresión para los sujetos oprimidos occidentales dentro de la Zona del No Ser que no compara con el acceso a derechos humanos/civiles/laborales, las normas de civilidad y los discursos emancipatórios reconocidos y vividos por los sujetos occidentales oprimidos dentro de la Zona del Ser.

Por otro lado, se hace referencia a la colonialidad de poder en la Interseccionalidad en jerarquías globales o vectores de discriminación con imbricaciones múltiples y heterogéneas. A continuación se explica cada una de las jerarquías, según Sousa y Meneses (2014) siguientes:

-División Internacional del Trabajo: Esto ocurre en el centro y en la periferia donde el capital organiza el trabajo en la periferia alrededor de formas represivas y autoritarias.

Por su parte, explica una formación de clase global donde coexisten y se organiza una diversidad de forma del trabajo, esclavitud, semi-servidumbre, trabajo asalariado, producción de pequeñas mercancías, entre otros, como fuente de producción de plusvalía mediante la venta de mercancías para obtener ganancias en el mercado mundial.

-El Sistema Interestatal del Trabajo: Constituye las organizaciones político-militares controladas por hombres europeos e institucionalizadas en administraciones coloniales y 


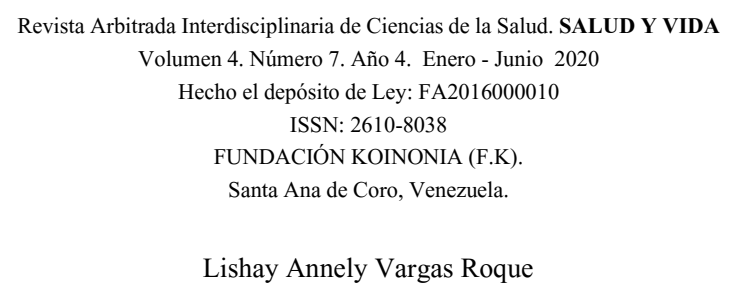

neocoloniales. En este sistema sigue floreciendo el colonialismo y la visión occidental en las personas que dirigen las instituciones de salud.

-Jerarquía Etno-racial Global: Se privilegia a las personas occidentales sobre las no occidentales. En relación a las instituciones de salud, se valora lo occidental, lo científico, mientras que las alternativas autóctonas, los saberes ancestrales para el mundo occidental no son aceptados.

-Patriarcado Xtriandad: Se da la primacía a los hombres sobre las mujeres y al patriarcado europeo sobre otras formas de relaciones de género. La medicina occidental está por encima de la medicina tradicional u otras formas de medicina.

-Jerarquía Epistémica Global: Se privilegia el conocimiento y la cosmología occidentales sobre el conocimiento y la cosmología no occidentales, institucionalizada en el sistema universitario en general. En este caso tiene más valor y peso el conocimiento de la medicina occidental que la medicina surgida de forma ancestral, autóctona, de boratios, la natural, la Homeopática y la china de los saberes transmitidos de generaciones en generaciones en los pueblos.

-Jerarquía Pedagógica Global: Se privilegia las pedagogías occidentales de matriz cartesianas sobre las pedagogías no occidentales, institucionalizada en el sistema escolar mundial. Sin embargo, las pedagogías de la medicina ancestral encontradas en las comunidades son inferiorizadas por el mundo occidental.

-Jerarquía Lingüística Global: La existencia de lenguas que deben ser reconocidas legal y oficialmente superiores a otras lenguas. Entre las lengua europeas y las no europeas que hacen primar la comunicación y la producción teórica, de conocimiento en la primeras, subalternizando las últimas como productoras de Folclore o de cultura, pero no de conocimiento, ni de teoría. En este sentido, no son reconocidas las lenguas que tienen un conocimiento distinto al occidental, en este caso particular el lenguaje no occidental de medicina tradicional, ancestral no son aceptados.

-Jerarquía Estética Global: Se privilegian las formas de belleza y gustos occidentales, mientras se inferiorizan las formas de belleza y gustos no occidentales, industrializados 


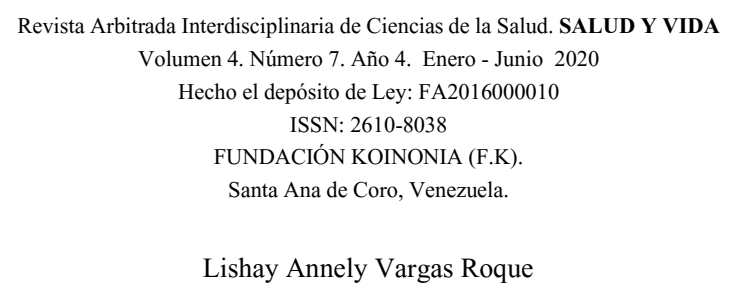

en los Ministerios de cultura y en la jerarquía de museos y galerías globales, así como en los diseños mercantiles industriales, se privilegia los espacios de las instituciones de salud occidentalizadas, no existe el sentido de pertenencia. La estética no occidentalizada se promueve en los espacios donde se desarrolla este encuentro de las comunidades con la medicina tradicional, la salud y la vida.

-Jerarquía Religiosa Global: Se da primacía a los cristianos sobre las espiritualidades no cristianas/no occidentales, institucionalizada en la globalización de la Iglesia cristiana católica, y luego el surgimiento de la protestantes. En este caso, la religión ocupa un lugar muy importante para la sanación desde lo espiritual de las personas en las comunidades. -Jerarquía Mediática Global: Existe el privilegio de aparatos de comunicación controlados en conjunto con los profesionales de la comunicación por occidente. Los medios utilizados por las instituciones de salud con su visión occidental, requieren de más humanidad en las personas que los manejan, más acercamiento hacia las comunidades desde la interculturalidad en salud y la pluriversidad. En la línea del no ser es necesario dejar un lado la comunicación mediática y que los medios den a conocer esa visión otra de la salud a través de esa mirada otra, valorando la medicina tradicional desde otra mirada otra de la salud, tomando en cuenta lo ancestral de los pueblos.

-Jerarquía de la Temporalidad: La edad para el mundo occidental privilegia la edad adulta productiva entre 16 y 64 años, inferiorizando y haciendo dispensable a los adultos mayores, a los niños y niñas.

Por otro lado en esta temporalidad, desde la no occidentalidad se toma en cuenta los saberes, independiente de la edad, visibilizando los saberes de las personas en sus comunidades con una visión intercultural.

-Jerarquía de la Cartografía Global: Establece la ubicación geográfica y espacial del ser humano occidentalizado y no occidentalizado en el contexto social. Estas personas las encontramos en las instituciones de salud y en las comunidades.

En esta investigación sobre la Interculturalidad como Direccionamiento Estratégico para la Visibilización de la Pluriversidad en Salud, aplica la interseccionalidad en la Línea de 


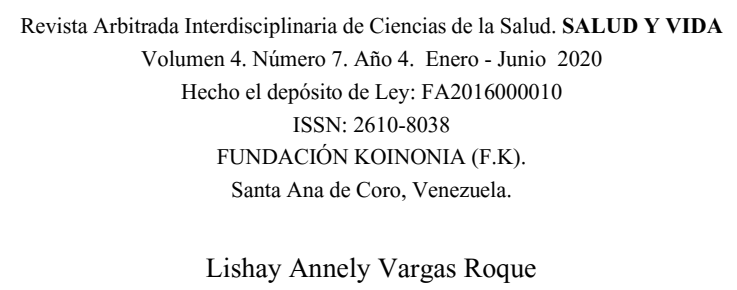

Frantz Fanón, en este caso para develar la colonialidad e interpretar las prácticas en la salud, las luchas que surgen tanto en la Zona del Ser y la del No Ser, tomando en cuenta la Línea de lo Humano. En este sentido, la pluriversidad en salud en el contexto de enunciación se realizó a través del diálogo que se sostuvo con las personas que realizan sus prácticas de salud en las comunidades y con directivos de Secretaría de Salud del Estado Falcón y Dirección Regional de Misión Barrio Adentro venezolana, para ello se utilizó la línea de Frantz Fanón. (Figura № 1).

Figura №1: El Sistema Público Nacional de Salud desde la Perspectiva de la Interseccionalidad en la Línea de Frantz Fanón

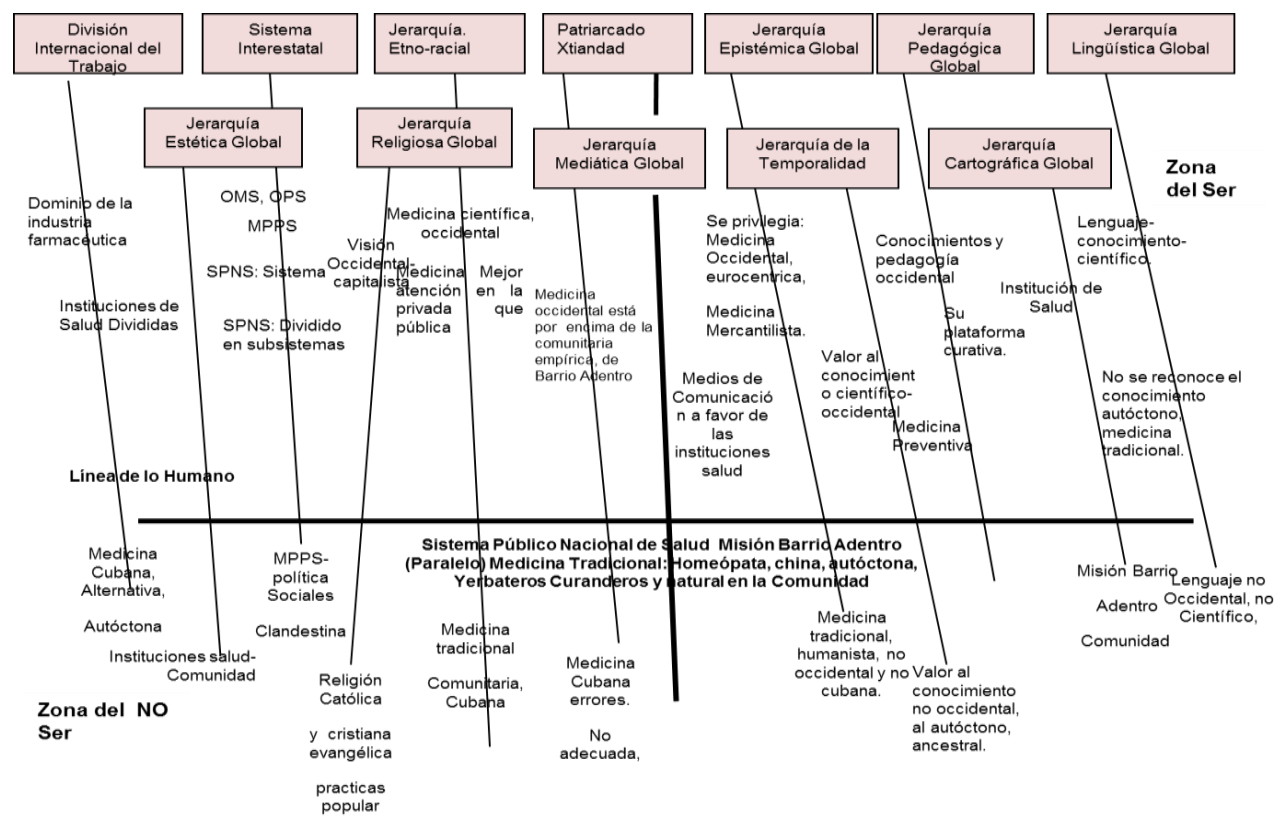

Fuente: Autora, 2019

\section{Nomenclatura:}

Medicina Científica/ occidental/eurocéntrica/colonial/mercantilista.

Medicina SPNS/Instituciones de Salud/ occidental/subsistema de salud/capitalista.

Medicina Barrio Adentro/no occidental/ preventiva/cubana/comunitaria.

Medicina Barrio Adentro/no occidental/preventiva/venezolana/comunitaria.

Medicina autóctona/nooccidental/comunitaria. 


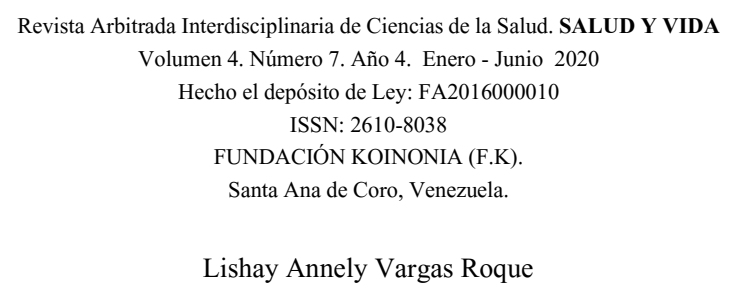

Medicina curanderos/no occidental/empírica/comunitaria. Medicina de Boratios/nooccidental/empírica/comunitaria. Medicina Homeopática/no occidental/religiosa/ comunitaria.

\section{ANÁLISIS}

Desde la perspectiva de la Interseccionalidad en la Línea de Frantz Fanón, el Sistema Público Nacional de Salud, la medicina occidental, es un modelo médico hegemónico, la salud es un negocio, es un sistema científico, la persona es vista como un paciente, se excluye a otros modelos, se ubica en la Zona del Ser. En la Zona del No Ser ubicamos a las Instituciones de salud de la Misión de Barrio Adentro que está en el Sistema Público Nacional de Salud por estar concebido como paralelo al tradicional, es no completamente aceptado, considerado con errores, cuestionada, la que vino a sustituir la medicina y al médico venezolano. A través del análisis de esta línea se visualiza el dominio que existe en el Sistema Público Nacional de Salud de las instituciones de salud, el racismo, la lucha de poderes en la cual está inmersa la salud. Así como también, de quienes dirigen las instituciones de salud no existe una valoración de las Instituciones de Salud por quienes dirigen estos centros, en el caso de quienes están en la Zona del Ser.

Esta medicina en la línea del No Ser, que es occidentalizada, fue incorporada por una nueva política de salud por el estado, para intervenir la salud del pueblo no sólo allí en las estructuras de salud, sino en la comunidad, en la casa, en la forma de organización de las personas, en su entorno social. Por otra parte, visualiza las instituciones de salud como un subsistema, cada quien por su lado, el modelo viejo no termina de morir y el nuevo no termina de nacer (Henry Suarez, diálogo, Mayo 28, 2018). La Misión Barrio Adentro nace para romper con los modelos hegemónicos impuestos por el neocapitalismo y llegar a los lugares más apartados del país. En esta Zona del No Ser, la persona es vista como un consultante, encontramos a los yerbateros y a la Medicina Milenaria. 


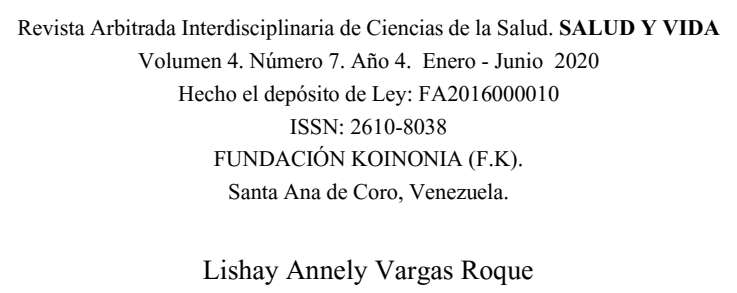

En este sistema el personal de salud, la medicina aplicada por los curanderos, boratios y homeópatas es una medicina empírica, no es ciencia, sin sustento científico, a la cual no se debería consultar según los criterios de la zona del ser. La medicina científica de las Instituciones de Salud es aceptada en la Zona del ser, que de acuerdo a la División Internacional del Trabajo es una medicina dominada por la industria farmacéutica, mientras por el Ministerio del Poder Popular para la Salud, desde el punto de vista de la Zona del Ser, es aceptado lo occidental.

En cambio, si vamos a la Zona del No ser la Misión Barrio Adentro es aceptada y es una política de salud para el estado venezolano, Jerarquía que representa el Sistema Interestatal del Trabajo. Así mismo, se presenta un Sistema Público Nacional de Salud, dividido en varios subsistemas, cada quien va por su lado, a pesar de que a nivel nacional se habla de un Sistema Púbico único de Salud, que está fraccionado, situación que se presenta de igual forma en el Estado Falcón, en el cual la medicina occidental tiene la visión de que la Misión Barrio Adentro vino a sustituir a las instituciones de Salud occidentales y a sus médicos.

En relación a la Jerarquía etno-racial, la medicina de los curanderos, boratios, autóctona es una medicina no comprobada, no válida, y estudiada para ser aplicada por la Zona del no Ser. En cambio, la medicina occidental ubicada en la Zona del Ser está por encima de esta medicina, es controlada por la colonialidad. La Misión Barrio Adentro a pesar que está formando parte del Sistema Público Nacional de Salud es vista de forma paralela al sistema tradicional en la Zona del Ser.

En cuanto a la Jerarquía Patriarcado $X$ triandad, la medicina occidental está por encima de la medicina de Barrio Adentro, la Homeópata, la medicina de los Yerbateros y de la autóctona en la Zona del Ser. Pero en la Zona del No ser es la medicina que las instituciones de salud sugiere que no sean consultadas, pero estas son aceptadas por las personas en las comunidades. Con la Jerarquía Epistémica Global, es mejor valorado el conocimiento de la medicina occidental que la medicina surgida de forma no 


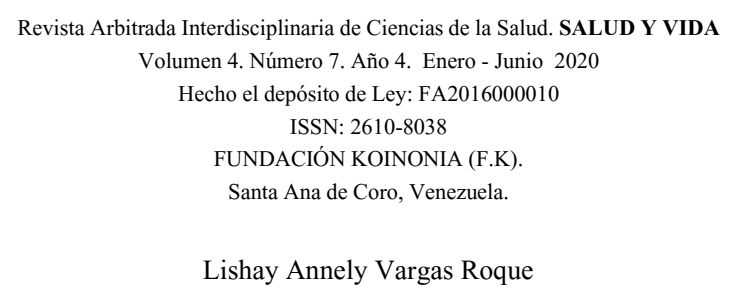

comprobada, autóctona y de boratios y de los saberes ancestrales, transmitidos de generaciones en generaciones.

Por otro lado la Jerarquía Pedagógica Global, las formas de producción de ese conocimiento en el Sistema Público de Salud la pedagogía en la Zona del Ser es dominada por las formas pedagógicas occidentales, y la Zona del No Ser privilegia a las pedagogías de la medicina de Misión Barrio Adentro, la autóctona, la de los Yerbateros y la autóctona. La Jerarquía del Lenguaje utilizado por este conocimiento de la Misión Barrio Adentro, los curanderos y de los boratios es subalternizado en la Zona del Ser. En cambio, en la Zona del No ser este conocimiento utiliza una lenguaje que valora ese conocimiento que no está comprobado y de esta Misión de Barrio Adentro para promover el bienestar de las comunidades en el hogar de las familias y en la organización comunitaria y social.

Con la Jerarquía estética global, ubicamos las condiciones en las cuales se ofrece la salud a las personas en las instituciones de salud occidental, condiciones que en la Zona del Ser, la atención es mejor ofrecida en las instituciones de salud privadas. En las instituciones de salud gubernamental occidentalizada existen limitaciones desde los materiales para el funcionamiento de estas por la corrupción, la falta de ética y moral de quienes la dirigen, la falta de gestión por los responsables de estas instituciones. Sin embargo, se manifiesta en esta Zona del Ser que la medicina occidental está por encima de la medicina de Barrio Adentro, de los curanderos, yerbateros, de la china, Homeopática y natural. En la Zona del No Ser ubicamos, a la Misión Barrio Adentro con sus estructuras idóneas, equipos y trato a sus usuarios de forma más humanistas, cercana al usuario en su hogar, en su comunidad. En este sentido, en general los médicos que dirigen estas instituciones de la Misión de Barrio Adentro, en estos últimos tiempos han sufrido una falta de apoyo por quienes dirigen las instituciones de salud en el estado, por el sólo hecho de no creer en esta política de salud. En cambio, esta forma institucional y política de Misión Barrio Adentro y de atención de la medicina Homeópata, de los 


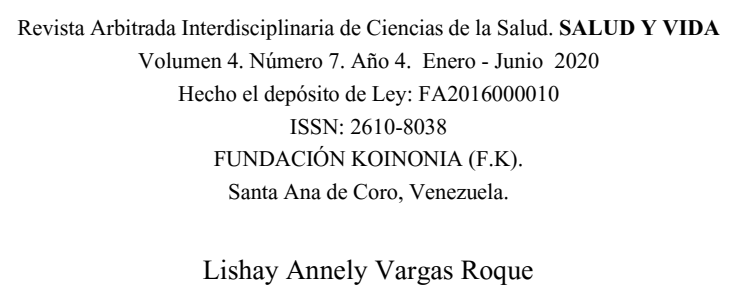

curanderos, Boratios, autóctona, la natural no es aceptada en la Zona del Ser, por no ser comprobada científicamente.

En la Jerarquía Religiosa se observa en la Zona del Ser, una medicina occidental, que no utiliza la religión para la atención y sanación de sus pacientes, se busca la sanación del paciente desde lo biológico, lo religioso interviene para proveer fe, esperanza a los familiares. Así mismo, las prácticas populares no son concebidas para la Medicina Occidental, para la sanación de las personas. En la Zona del No Ser, encontramos que los curanderos, Boratios, Homeópatas y a la medicina china utilizan la religión para que la persona alcance su sanación a través de su acercamiento a Dios, ya sea a través de la religión católica o de la religión cristiana evangélica. En cuanto a Misión Barrio adentro, no utiliza la religión para que las personas puedan alcanzar la salud de sus pacientes. Es importante resaltar que en la Zona del No Ser la sanación se enfoca desde lo espiritual del ser humano. En el caso de las prácticas populares del pueblo falconiano no es aceptado en lo religioso en la Zona del No Ser, pero es aceptado por los pueblos.

Por otro lado la Jerarquía Mediática Global, las instituciones de salud con una visión occidental controlan estos medios a su favor, en las cuales existe poco acercamiento hacia las comunidades, en lo referente a la Zona del Ser. En la Zona del No Ser los medios no son utilizados para la promoción de la salud, el acercamiento a las comunidades, en pro de mejorar el bienestar y promover los saberes, culturales de los pueblos. A diferencia de la Zona del Ser, existe un interés por los que están ubicados en la Zona del Ser, se dan a conocer con este encuentro los saberes para mejorar la salud de las personas en lo familiar y social. Sin embargo, es importante señalar que en estos últimos tiempos los medios de comunicación no son utilizados para promover las políticas y programas de las instituciones de salud, lo cual permitiría un mejor conocimiento a la población venezolana sobre las políticas de salud y de integración a las organizaciones comunales en las comunidades con su participación protagónica de bienestar integral en lo familiar y comunitario. 


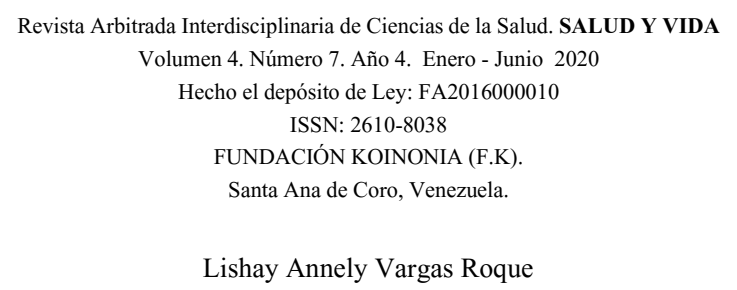

En la Jerarquía de la Temporalidad, se valora en la Zona del ser y se privilegia los conocimientos científicos comprobados, mientras que en la Zona del No Ser se valora los saberes culturales transmitidos de generación en generación, los conocimientos empíricos, visibilizándolos en pro del bienestar de las personas, logrando la salud de las comunidades. Por su parte, en la Jerarquía de la Cartografía se ubica al profesional de la salud en un enfoque propio de las Instituciones de Salud Occidentales, en el caso de la Zona del Ser. Pero en la Zona del No ser se ubica en la comunidad y las personas con sus saberes aplicados privadamente a lo personal y familiar en pro del bienestar de las salud.

\section{REFERENCIAS CONSULTADAS}

1. Dussel, E (2009) Filosofía de la Liberación y Diálogo Intercultural. Vol № 47, pp 107-122.

2. Dussel, E. (S/A) Sistema Mundo y Transmodernidad.

3. Fanon Frantz (1983) Los Condenados de la Tierra. Séptima reimpresión. Editorial Fondo de Cultura Económica, México.

4. Fanon Frantz (1983) Los Condenados de la Tierra. Séptima reimpresión.

5. Fanón, Frantz (2010) Piel Negra, Mascaras Blancas. Askal: Madrid, España.

6. Grosfoguel, Ramón (2011) La Decolonización del Conocimiento: Diálogo Crítico entre la Visión Decolonial de Fanon y la Sociología Decolonial de Boaventura de Sousa Santos. Editorial Fond

7. Grosfoguel, Ramón y Castro, G Santiago (2007) El Giro Decolonial. Siglo de Hombres Editores.

8. Grosfoguel, Ramón (2011) Decolonizing Post-colonial-economy: Post conlonial studies and paradigms of political- economy: Transmodernity, Decolonial Thinking and Global Coloniality, transmodernity: Journal of Peripheral cultural. Production of the Luso-Hispanice.World. Vol 1,№1, p.1-38. 
Revista Arbitrada Interdisciplinaria de Ciencias de la Salud. SALUD Y VIDA

Volumen 4. Número 7. Año 4. Enero - Junio 2020

Hecho el depósito de Ley: FA2016000010

ISSN: $2610-8038$

FUNDACIÓN KOINONIA (F.K)

Santa Ana de Coro, Venezuela.

Lishay Annely Vargas Roque

9. Grosfoguel, Ramón y Castro, G Santiago (2007) El Giro Decolonial. Siglo de Hombres Editores.

(C2020 por los autores. Este artículo es de acceso abierto y distribuido según los términos y condiciones de la licencia Creative Commons Atribución-NoComercial-Compartirlgual 4.0 Internacional (CC BY-NC-SA 4.0)

(https://creativecommons.org/licenses/by-nc-sa/4.0/). 